\title{
Revolución Bolivariana: políticas culturales en la Venezuela Socialista de Hugo Chávez (1999-2013)
}

\author{
Bolivarian Revolution: Cultural Policies in the Socialist \\ Venezuela of Hugo Chávez (1999-2013) \\ Revolução Bolivariana: políticas culturais na Venezuela \\ socialista do Hugo Chávez (1999-2013)
}

\section{Gisela Kozak Rovero}

UNIVERSidAd CENTRAL DE VENEZUELA, CARACAS, VENEZUELA

Profesora titular de la Escuela de Letras, de la Maestría en Estudios

Literarios y de la Maestría en Gestión y Políticas Culturales de la

Universidad Central de Venezuela, Caracas. Doctora en Letras por la Universidad Simón Bolívar, Caracas. Autora de: Rebelión en el Caribe

Hispánico. Urbes e historias más allá de boom y la postmodernidad

(Ediciones La Casa de Bello, 1993); La catástrofe imaginaria (Planeta-

Celarg, 1998); Venezuela, el país que siempre nace (Alfa, 2008); y de los textos de ficción Pecados de la capital (Monte Ávila, 2005), En rojo (Alfa, 2011), Latidos de Caracas (Alfaguara, 2007) y Todas las lunas (Equinoccio, 2011; Sudaquia, 2014).Tiene numerosos artículos en revistas especializadas. Correo electrónico: gisela.kozak@gmail.com 


\section{Resumen}

El artículo presenta un balance de las políticas culturales venezolanas a partir de la definición de cultura y de las áreas de gestión tipificadas por UNESCO. Se toma en cuenta para el análisis la información oficial e investigaciones independientes acerca de cuatro aspectos sustantivos: a) Institucionalidad Cultural, Legislación, Inversión Cultural y Públicos para la Cultura; b) Creadores, Gestores, Cultores e Intelectuales en la Venezuela de la Polarización; c) El Hombre Nuevo, la Mujer Nueva: Objetivos Estratégicos de la Revolución Bolivariana; d) Las deudas históricas con el sector cultural.

Palabras clave: políticas culturales; democracia; institucionalidad cultural; campo cultural; socialismo; Venezuela

\section{Abstract}

The article gives an overview of Venezuelan cultural policies from the definition of culture and cultural activities typified by UNESCO. It analyzes official information and independent research about four substantive issues: a) Cultural Institutions, Legislation, Cultural and Public Investment for Culture; b) Creators, Cultural Managers, "Cultores" and Intellectuals in Venezuela; c) The New Man, New Woman: Strategic Objectives of the Bolivarian Revolution d) The historical debts to the cultural sector.

Keywords: cultural policies; democracy; cultural institution; cultural field; socialism; Venezuela

\section{Resumo}

$\mathrm{O}$ artigo dá uma visão geral das políticas culturais venezuelanas a partir da definição de cultura e atividades culturais da UNESCO. É uma análise de dados oficiais e de pesquisa independente sobre quatro questões de fundo: a) Instituições Cultural, Legislação, Investimento, Público para a Cultura; b) Artistas, Gestores, "Cultores" e intelectuais na Venezuela de polarização; c) $\mathrm{O}$ Novo Homem, Nova Mulher: Objetivos Estratégicos da Revolução Bolivariana d) As dívidas para o setor cultural.

Palavras-chave: políticas culturais; democracia; instituições culturais; campo cultural; socialismo; Venezuela

RECIBIDO: 2 DE MAYO DE 2014. APROBADO: 23 DE MAYO DE 2014. DISPONIBLE EN LÍNEA: 15 DE ENERO DE 2015

\section{Cómo citar este artículo:}

Kozak Rovero, Gisela. "Revolución Bolivariana: políticas culturales en la Venezuela Socialista de Hugo Chávez (1999-2013)". Cuadernos de Literatura 19. 37 (2015): 38-56. doi:10.11144/Javeriana.CL19-37.rbpc 


\section{Criterios para un Balance}

La Organización de las Naciones Unidas para la Educación, la Ciencia y la Cultura (UNESCO) define la cultura como "el conjunto de los rasgos distintivos, espirituales, materiales y afectivos que caracterizan una sociedad o grupo social. Ella engloba, además de las artes y las letras, los modos de vida, los derechos fundamentales del ser humano, los sistemas de valores, creencias y tradiciones" (UNESCO, 1). La cultura es la sangre misma de nuestras vidas como individuos en relación con el mundo que nos rodea y así ha sido a lo largo de la historia y en todos los rincones del mundo. En los Estados nacionales modernos es usual que existan políticas destinadas a reforzar aquellas prácticas culturales que se consideran significativas para sus habitantes. Cuando se trata de democracias tal reforzamiento jamás debe confundirse con el condicionamiento o la dirección de los procesos creativos y sociales de recepción, producción y transmisión cultural. La sola existencia de tantos hombres y mujeres cuya obra literaria, visual, teatral, musical, intelectual, de animación y de gestión - por no hablar de la defensa de sus idiomas, ideas políticas o convicciones sobre el tema religioso- se ha realizado al margen o en contra de los grandes poderes en juego, es un ejemplo de los límites precisos de la acción estatal en este terreno. En una democracia, la libertad de expresión, pensamiento y creación asegura que la diversidad de registros, expresiones y formas de vida puedan tener existencia en el Estado nacional; visto así, las políticas culturales deberían acompañar y apoyar la investigación, conservación, financiamiento, administración, estímulo a la creación o difusión en áreas como:

- Patrimonio cultural (Protección del Patrimonio Mundial $)^{1}$

- Textos impresos y literatura

- Música

- Artes escénicas

1 Según el artículo I de esta convención se considera patrimonio cultural:

"-Monumentos: obras arquitectónicas, de escultura o de pintura monumentales, elementos o estructuras de carácter arqueológico, inscripciones, cavernas y grupos de elementos, que tengan un valor universal excepcional desde el punto de vista de la historia, del arte o de la ciencia, - Los conjuntos: grupos de construcciones, aisladas o reunidas, cuya arquitectura, unidad e integración en el paisaje les dé un valor universal excepcional desde el punto de vista de la historia, del arte o de la ciencia,

-Los lugares: obras del hombre u obras conjuntas del hombre y la naturaleza así como las zonas, incluidos los lugares arqueológicos que tengan un valor universal excepcional desde el punto de vista histórico, estético, etnológico o antropológico".

En el caso de este trabajo, nos referimos al patrimonio cultural nacional. 
- Artes plásticas

- Cine y fotografía

- Radio y televisión

- Actividades socioculturales (Recomendación sobre la Normalización) ${ }^{2}$

- Gastronomía y turismo cultural

- Antropología, Historia, Sociología, Crítica

Cada uno de estos temas exige estudios específicos que sobrepasan la extensión de este artículo y el área de competencia de quien lo escribe; asimismo, la gestión regional y municipal posee características particulares que requieren análisis especializados. Un balance ideal de la gestión cultural del Estado en la Venezuela del siglo XXI requeriría de un inmenso esfuerzo capaz de tomar en consideración estos aspectos y otros igualmente vitales como las propuestas estéticas surgidas al calor de estos años tan difíciles para Venezuela, la actividad cultural en todo el país (a la que Caracas injustamente le hace sombra en cuanto a proyección), y aspectos más propiamente técnicos relativos a cifras presupuestarias y de gestión. Dada la imposibilidad presente de realizar un estudio de esta naturaleza, las siguientes páginas serán orientadas al balance de la gestión estatal de la cultura en los últimos catorce años en función de cuatro aspectos: 1) Institucionalidad Cultural, Legislación, Inversión Cultural y Públicos para la Cultura; 2) Creadores, Gestores, Cultores e Intelectuales en la Venezuela de la polarización; 3) El hombre nuevo, la mujer nueva: objetivos estratégicos de la Revolución Bolivariana; 4) Balance: $\mathrm{i}$ se han saldado las deudas pendientes con el sector cultural en 1999 ?

\section{Institucionalidad cultural, legislación, inversión cultural y públicos para la cultura}

Para el año 1999 Venezuela contaba con numerosas instituciones culturales financiadas por el Estado:

2 La Recomendación sobre la normalización internacional de las estadísticas relativas al financiamiento público de las actividades culturales define las siguientes actividades como socioculturales:

"7.0 Animación sociocultural, casas de la cultura y fomento de las actividades de aficionados

7.1 Asociaciones cívicas o profesionales

7.2 Otras actividades socioculturales (ceremonias, prácticas sociales asociadas a las creencias religiosas, morales, éticas o filosóficas)

7.3 Formación fuera de los sistemas de enseñanza oficial

7.4 Otras actividades necesarias para las actividades socioculturales" 
- Monte Ávila Editores

- Biblioteca Ayacucho

- La Casa de Bello

- Instituto Autónomo Biblioteca Nacional

- Imprenta Nacional

- Museos: Arte Contemporáneo, Ciencias y de Bellas Artes, Lía Bermúdez, Galería de Arte Nacional, Alejandro Otero, Arte Contemporáneo del Zulia, Jacobo Borges, Jesús Soto, Arturo Michelena, Arte Popular de Trujillo, Arte Popular de Petare, entre otros.

- Festival Internacional de Teatro

- Centro Nacional de Cinematografía

- Compañías Nacionales de Teatro y Danza

- Movimiento de Teatro Penitenciario

- Complejo Cultural Teresa Carreño

- Consejo Nacional de la Cultura

- Fundarte

- Centro de Estudios Latinoamericanos Rómulo Gallegos

- Centro Cultural La Estancia

- Sistema Nacional de Orquestas y Coros Juveniles e Infantiles de Venezuela

- Premio Internacional de Novela Rómulo Gallegos

- Festival de Teatro de Oriente

- Direcciones de Cultura de la universidades públicas

- Televisora Nacional Canal 5, Venezolana de Televisión y Radio Nacional de Venezuela

- Sistema de coros de la Schola Cantorum

- Ateneos y casas de la cultura en todo el país

- Espacios públicos como plazas, paseos y bulevares

- Salas de cine y teatro

- Red de librerías Kuai Mare

- Direcciones de Cultura de alcaldías y gobernaciones

- Consejo Nacional de la Cultura

- Archivo General de la Nación

- Fundación Vicente Emilio Sojo

- Cameratas Barroca y Renacentista

- Revista Imagen, Nacional de Cultura y Encuadre, entre otras.

- Grupos de teatro y danza contemporáneos que recibieron subsidios del Estado. 
Estas instituciones han sido conservadas con su nombre original o con otro nombre por el Gobierno revolucionario. Sobresale en esta gestión la creación del Ministerio del Poder Popular para la Cultura en el año 2005, cuyo primer ministro fue el arquitecto Francisco Sesto, y la organización de las plataformas culturales que se definen por actividades relacionadas como Cine y Audiovisual, Libro y Lectura, Pensamiento, Patrimonio y Memoria, Artes Escénicas y Musicales, Artes de la Imagen y Espacio. ${ }^{3}$

Otra novedad son los gabinetes estatales culturales, la Misión Cultura, orientada a la actividad cultural comunitaria en función de la consolidación de la "identidad nacional", la Licenciatura en Educación, mención Desarrollo Cultural, y el Ministerio del Poder Popular de Asuntos Indígenas, entre cuyas líneas está al reforzamiento y la protección cultural de estas comunidades. Se han fundado algunas instituciones nuevas. Entre las más conocidas encontramos:

- Editorial El Perro y la Rana

- Villa del Cine

- Distribuidora Nacional

- Centro Nacional de Historia

- Museo de Arquitectura

- Fundación Red de Arte

- Canales de televisión Ávila, Vive, Colombeia y TVES

- Radio ALBA FM

- Universidad de las Artes (UNEARTES)

- Sistema Masivo de Revistas: A Plena Voz, La Roca de Crear, Así Somos, Se Mueve, Arte de Leer, Memorias de Venezuela, Buen Vivir y La Revuelta

En cuanto a recursos financieros, ha existido una importante inversión cultural que no se refleja mucho en el presupuesto oficial, cuya asignación al sector no supera el porcentaje promedio anterior a 1998; por ejemplo, las transferencias hechas por otras vías como los créditos adicionales, las erogaciones especiales para proyectos específicos como el Mausoleo del Libertador y las contribuciones de otros ministerios, como es el caso del Ministerio del Poder Popular para el Despacho de la Presidencia y Seguimiento de la Gestión de Gobierno respecto al Sistema Nacional de Orquestas y Coros Juveniles e Infantiles de Venezuela. En todo caso, vale la pena observar, según datos de Carlos Enrique Guzmán

3 Ministerio del Poder Popular para la Cultura. Organigrama Estructural Abril 2010. Caracas: Ministerio del Poder Popular para la Cultura, 2010. 
Cárdenas, la evolución de la inversión pública en cultura en el país. ${ }^{4}$ Según este autor, el presupuesto oficial para el sector cultural nunca ha excedido el 0,53\% del presupuesto nacional (7).

El financiamiento del sector cultural se enmarca en la política de distribución de la renta petrolera característica de la revolución bolivariana. Destacan el apoyo sostenido al Sistema Nacional de Orquestas Infantiles y Juveniles y al cine nacional tanto como la producción masiva de impresos, discos y materiales audiovisuales. Habría que sumar los eventos públicos (desde comunitarios hasta grandes festivales de poesía, conciertos de música clásica y encuentros internacionales de intelectuales), amén del respaldo otorgado a la cultura popular tradicional, urbana y aborigen, a la animación cultural juvenil y a las muy diversas formas del arte de calle. El problema es que no disponemos de encuestas de consumo cultural confiables y sistemáticas que den cuenta de la creación de nuevos públicos para la cultura acordes con la gigantesca cantidad de objetos producidos, la gratuidad o bajo costo de dichos eventos y la presencia de la gestión cultural estatal en las bases de la sociedad durante los 14 años de gestión cultural del Gobierno revolucionario. Las excepciones serían los estudios sobre el comportamiento lector del Centro Nacional del Libro (CENAL), las cifras relativas a espectadores del cine venezolano (Centro Nacional de Cinematografía), y el número de niños y jóvenes participantes en el Sistema Nacional de Orquestas y Coros Juveniles e Infantiles de Venezuela según la propia institución.

Igualmente se manejan algunas cifras de impacto poblacional en las Memoria y Cuenta del Ministerio del Poder Popular para la Cultura desde 2005 hasta 2012 - disponibles en su página Web oficial- pero esos números no pueden compararse con estudios de consumo cultural amplios, sistemáticos y cuidadosos como el "Estudio de Consumo Cultural del Proyecto Pobreza", de la Universidad Católica Andrés Bello, cuyos resultados nos son halagüeños para la gestión cultural gubernamental pero que sólo cubre hasta el año 2008. Las cifras de estudio nos indican, por ejemplo, que para esa fecha el $85 \%$ de los entrevistados no visitaba museos y galerías de arte, el $91 \%$ no asistía a conciertos de música clásica y el $75 \%$ tampoco a conciertos de música popular; $69 \%$ de la población no entraba nunca a una librería y el $74 \%$ tampoco a una biblioteca. Estos porcentajes

4 Carlos Enrique Guzmán Cárdenas. La Inversión Pública Cultural en Venezuela 1965-2011. Caracas: Observatorio Venezolano de Políticas Culturales-Informe de Investigación 1-SIPECC/ GCIECC-02-2011, 2011. 
coinciden con los sectores económicamente menos favorecidos, gran objetivo en principio de la gestión cultural oficial.

No contamos hoy con un instrumento semejante sobre el consumo cultural propio de los organismos oficiales en el período comprendido entre $1999 \mathrm{y}$ 2013, ni siquiera después de la fundación del ministerio en el año 2005, excepto, como ya se dijo, en lo relativo a la lectura. Vale la pena analizar estas cifras porque dan una idea de la incidencia de las políticas del Estado en el público lector del país: la inversión no es proporcional con el impacto en los hábitos lectores, sin negar la magnitud del esfuerzo y que se han facilitado libros a los públicos tradicionales universitario y profesional. En las siguientes líneas sólo se tomará en cuenta la lectura de libros y se dejará a un lado la de otro tipo de material. La Encuesta de Consumo Cultural de la UCAB ya mencionada indica que el $48 \%$ de la población en 2008 leía libros y, por su parte, el "Estudio del Comportamiento Lector, Acceso al Libro y la Lectura en Venezuela 2012", efectuado por el Centro Nacional del Libro (CENAL), indica que 10.503 .780 personas (50\% de la población alfabetizada) leen al menos un libro. La variación en estos últimos años ha sido entonces de un 2\%. Si se contrasta el estudio del CENAL con análisis publicados en el año 2013 como "Venezolanos comelibros", de Lisseth Boon y Jesús Alberto Yajure, y "El libro: entre cuentos y cuentas", de Carlos Delgado Flores, constatamos que:

- El público lector de libros ha aumentado lentamente en los últimos años.

- Venezuela ha triplicado el número de títulos publicado en el país por habitante: de 4 títulos al año 2004 ha pasado a 12 en el año 2012. De hecho, según el Centro de Estudios del Libro de América Latina y del Caribe (CERLALC), el Estado venezolano es el segundo en América Latina en cuanto a producción de libros.

- De los 10.503.780 de los lectores que leen libros, la mitad no pasa de 4 libros al año y la cuarta parte de 10 en el mejor de los casos.

- En 2008 se importaron libros por un valor de 422 millones de dólares, y en 2011, 93 millones. Esta disminución obedece a las restricciones cambiarias impuestas por CADIVI.

- A pesar de los grandes tirajes gratuitos o a precio simbólico del gobierno nacional a través de la Plataforma del Libro y la Lectura, el 80 \% de los entrevistados por el CENAL afirma no haber obtenido nunca una publicación del Estado.

- Leemos un promedio del 2\% por encima de la media de América Latina.

- Ha crecido el interés en libros de tema histórico y político social. 
- De los diez libros más leídos sólo dos son venezolanos, Doña Bárbara, de Rómulo Gallegos, y Casas Muertas, de Miguel Otero Silva, ya posicionados entre los venezolanos mucho antes de 1998.

- La temática preferida por el público lector es la autoayuda y el género literario preferido es la novela, atendidos especialmente por el sector editorial privado. La intervención del Estado no ha modificado sustancialmente los hábitos mayoritarios de lectura anteriores a 1998.

Habría que agregar que los organismos estatales educativos y culturales no someten a la población escolar a mediciones internacionales y nacionales confiables capaces de validar si los millones de libros publicados por el Estado tienen impacto real en las habilidades de lecto-escritura, la elevación de la educación y el cultivo de las ideas y el conocimiento en la sociedad. Este tipo de política que produce libros pero no se cerciora concienzudamente de su aprovechamiento real tiene su expresión más patente en el proyecto editorial por excelencia de la revolución: El Perro y la Rana. Esta editorial ha publicado cientos de títulos en diversas colecciones a precios simbólicos. Aunque es muy loable su intención de publicar a autores noveles, las políticas de distribución, venta y difusión crítica y mediática de los poetas, narradores y ensayistas que comienzan su trayectoria convierten sus producciones en un gesto simbólico cuyo impacto real en el público lector no es significativo.

Por otra parte, la oferta de libros es limitadísima. Importar libros es muy costoso dado el control de cambio y el gobierno edita de manera caprichosa y con marcado sesgo ideológico, lo cual desde luego aleja a públicos no alineados. Este alejamiento tiene otra arista importante: la competencia con el sector privado es absolutamente desleal en detrimento del conocimiento de la obra de autores no oficialistas por los precios de los libros no subsidiados. Por ejemplo, no existe un programa de compras para bibliotecas que asegure la presencia de la diversidad editorial venezolana en todo el país; los autores no identificados con la revolución no cuentan pues con el apoyo del estado y sus recursos, que pertenecen a toda la población, para el conocimiento de su trabajo y la búsqueda de potenciales lectores.

Desde el punto de vista legal, tenemos que la Constitución de la República Bolivariana de Venezuela (1999) posee un articulado de avanzada respecto a la cultura en su Capítulo VI "De los Derechos Culturales y Educativos" y en el Preámbulo, pues consagra la creación y disfrute cultural en plena libertad en el marco de una nación pluricultural cuya diversidad esencial debe respetarse. No obstante, la Ley de Cultura que debería desarrollar estos principios constitucionales no ha sido aprobada todavía. El Proyecto de Ley de Cultura, sometido a 
primera discusión en el año 2009 y que está en la agenda legislativa del año 2013, no le hace honor a este articulado pues define la cultura venezolana en términos restrictivos y tradicionales como los de "culturas constitutivas de la nacionalidad" (indígenas, afrodescendientes, cultura criolla popular urbana y rural), sin contemplar como materia de la ley las tecnologías de información y comunicación, las industrias culturales (editoriales, música, radio y televisión de servicio público, etc.) y los aportes de las sucesivas oleadas de inmigrantes a las culturas que conviven en el territorio nacional. Otras leyes pendientes de aprobación definitiva son la Ley de Fomento y Protección al Desarrollo Artesanal y la Ley de Protección Social del Artista Venezolano, alineadas con el Plan Socialista Simón Bolívar (2007-2013) y el Plan de la Patria (2013-2019) más que con la Constitución vigente.

\section{Creadores, Gestores, Cultores e Intelectuales en la Venezuela de la polarización}

En las dinámicas culturales venezolanas se han producido complejos procesos de exclusión y autoexclusión por motivos políticos que han marcado todo el circuito de creación, difusión y recepción de la creación y el disfrute cultural. El financiamiento estatal de la cultura exige lealtades o por lo menos discreción política, situación muy frecuente en campos como la música, la danza o la cultura popular, y menos en la literatura, la edición, las artes visuales, el teatro, el pensamiento o el periodismo cultural. El cine es un capítulo aparte pues efectivamente la creación de la Villa del Cine y el financiamiento estatal para la producción y difusión han dado frutos, aunque tal como indicó hace años el ex Ministro del Poder Popular Para la Cultura Francisco Sesto, las personas no afectas a la revolución no deberían merecer el apoyo del Estado para su trabajo. Se perdió, además, el cine de tema político crítico con el poder del Estado tan floreciente en otra época. Hay que sumar a esta situación la cacería de brujas y la obligación de participar en el proselitismo político revolucionario que abruma al personal de las instituciones del Estado en general y del sector cultural en particular. En todo caso, los artistas, gestores, escritores, pensadores y técnicos abiertamente no identificados con el Gobierno nacional tenemos que desenvolvernos sin la ayuda del Estado, situación que, por cierto, nos ha brindado una autonomía y una conciencia de nuestra condición y responsabilidad que sin duda es un activo de cara al futuro, cuando la democracia no sea una formalidad electoral sino la forma de negociar en paz y progreso las diferencias entre los habitantes del país.

En Caracas, compañías como el Grupo Actoral Ochenta, SKENA y Hebu Teatro han tenido éxitos resonantes en salas privadas como el centro Trasnocho Cultural. La fundación de centros alternativos estilo Los Galpones y otras 
galerías de arte le han dado espacio a propuestas de artes visuales sin entrada en el Sistema de Museos. Es de destacar un movimiento musical como la Movida Acústica Urbana, con su original propuesta de reelaboración y recreación de la música popular tradicional venezolana, y la mudanza del Ateneo de Caracas a una nueva sede luego del generalizado secuestro de estos espacios a lo largo y ancho del país. En la capital y en otras regiones, los espacios autónomos de la cultura han tenido apoyo de alcaldías y gobernaciones de oposición en la medida de los recursos disponibles y dependiendo de la sensibilidad frente al tema cultural que posean las autoridades en ejercicio. En Caracas destacan la Dirección de Cultura de la Alcaldía de Chacao y los esfuerzos de las de Baruta, Sucre y El Hatillo. En el mundo editorial iniciativas privadas como ALFA Editorial, Editorial Libros Marcados, Libros de El Nacional y Lugar Común, entre otras, han permitido canalizar el interés por la política, la historia y la literatura venezolana que ha aumentado en el país en los últimos años y han sido la respuesta a los riesgos e inconvenientes de la importación de libros dado el control de cambio existente.

Sin duda, no ha habido actividad en la que la polarización política no se haya reflejado. El Gobierno ha fundado un Centro Nacional de Historia como contrapeso a la Academia Nacional de la Historia, que no obedece a sus designios, y a las universidades públicas autónomas que se han resistido a sus intentos de dominación. Se supone que este Centro rescatará una memoria histórica perversamente ignorada por la "historia oficial", cuando la realidad es que en Venezuela las universidades públicas han sido dominadas por la intelectualidad de izquierda y generaciones enteras de historiadores han revisado la historia venezolana desde perspectivas en las que, por cierto, el marxismo ha tenido un papel estelar. Es mentira que en Venezuela antes de 1999 se imponía una historiografía racista, acartonada, burguesa y antipopular que respondía a las políticas educativas y culturales de la derecha imperialista en el poder. La educación venezolana pública y privada no respondió a una sola lógica en todos sus niveles.

En el mundo editorial nos encontramos con la Plataforma del Libro y la Lectura conformada por Monte Ávila Editores, Editorial El Perro y la Rana, Biblioteca Ayacucho, Red de Librerías del Sur, Casa Nacional de las Letras Andrés Bello, Imprenta de la Cultura, Distribuidora Venezolana de la Cultura, Centro Nacional del Libro. El Estado escoge, edita, distribuye y vende, asunto que forma parte de la tradición de las políticas culturales del Estado venezolano. El problema es que un Gobierno que quiso someter a la población a una iniciativa como el fracasado Plan Revolucionario de Lectura, que incluyó selecciones de discursos del presidente fallecido Hugo Chávez y del expresidente cubano Fidel Castro, no está tan interesado en el estudio y en el libre debate como en 
el adoctrinamiento ideológico en términos revolucionarios. Por otra parte, las colecciones de ensayo político, histórico y social de editoriales estatales como El Perro y la Rana y Monte Ávila Editores, no reflejan en lo absoluto la diversidad política e ideológica de la sociedad venezolana. Aunque Monte Ávila Editores ha seguido publicando literatura venezolana, su colección insignia es Milenio Libre, línea dedicada a publicar lo más granado de la izquierda radical internacional. Monte Ávila Editores cuenta con un excelente fondo editorial de varias décadas y dos programas literarios, Biblioteca Básica de Autores Venezolanos y Autores Inéditos, muy positivos a pesar de las irregularidades en cuanto a distribución y promoción. Por fortuna, la Biblioteca Ayacucho ha conservado, en lo que cabe, su perfil de editorial dedicada al pensamiento y la creación latinoamericanos, pero no es la estrella de los proyectos editoriales del Gobierno.

En el caso del teatro, destaca la pérdida del subsidio por parte de grupos con extraordinaria trayectoria como Skena y el Grupo Actoral Ochenta, acción que marcó una forma de gestión visible en la división del mundo musical popular urbano y tradicional, notable en contrataciones y eventos públicos. Actores, directores y músicos se han visto forzados a cambiar de rumbo o a presentar espectáculos en salas privadas dada la desaparición de espacios para telenovelas y musicales en la televisión comercial, y el fuerte sesgo político partidista de canales como TVES, Vive TV, Colombeia, Ávila TV, los cuales responden a las líneas de orientación y segregación ideológica de las políticas culturales, educativas y comunicacionales del estado. Lo más preocupante es que los espacios independientes disminuyen porque la revolución trata de captarlos en su empeño hegemónico: bajar la cabeza o autocensurarse pueden ser las opciones.

Probablemente el caso más llamativo de la gestión del Gobierno, particularmente en su crítica a la perspectiva "elitista" de la gestión cultural anterior a 1999, es su tratamiento respecto a los museos. El Sistema Nacional de Museos está conformado por: Galería de Arte Nacional, Museo de Arte Contemporáneo, Museo de Bellas Artes, Museo de Ciencias, Museo Alejandro Otero, Museo de la Estampa y del Diseño Carlos Cruz-Diez, Museo Arturo Michelena, Museo de Arte Popular, Museo de Arquitectura, Museo de la Fotografía, Museo de Ciencia y Tecnología, Museo de Coro, Museo de Calabozo. María Elena Ramos, en La Cultura Bajo Acoso, hace un preocupante balance sobre los museos venezolanos en el siglo XXI, pues describe con precisión la estrategia seguida por el Gobierno nacional en términos de la pérdida del perfil propio de cada uno, evidente no sólo en la desaparición de sus logotipos y en el cambio de sus líneas de exposición e investigación, sino también en la pérdida de autonomía al centralizar su administración a través de la Fundación Museos Nacionales. El rechazo a las ex- 
posiciones de artistas individuales, el cuestionamiento a la figura del curador y el desprecio por el personal calificado de custodia y preservación de las colecciones de cada institución marcó, según Ramos, especialmente la gestión del exministro Farruco Sesto, decidido a acabar con el museo como instrumento pedagógico y de la memoria nacional a favor de un ideal popular nunca definido más allá del "anti-elitismo" y de la instrumentación partidista de las políticas respecto al sector. Debo agregar entre paréntesis que hasta de refugios para damnificados han servido museos como el Alejandro Otero, en abierto irrespeto a estas personas albergadas en un sitio no apto para tal fin y en un acto de confiscación del patrimonio de la nación que pertenece a cada hombre y mujer venezolanos.

Continuando con el balance de Ramos, ni siquiera la inauguración de nuevos museos o de la sede de la Galería de Arte Nacional compensan los reales peligros que enfrenta nuestro patrimonio artístico, pues se ha pretendido incluso sustraer las colecciones de cada museo en un afán centralizador que atraviesa todos los niveles del Gobierno, en todas las áreas. Durante la gestión del exministro Pedro Calzadilla se apreció una disminución de la hostilidad contra las instituciones museísticas y se reconoció la necesidad de volver a las muestras individuales; no obstante, Calzadilla aupó el uso de los museos en conmemoración de los 20 años del golpe de Estado del año 1992. Ramos menciona los siguientes eventos: Testimonios de un Tiempo Político (Museo de Bellas Artes); El Camino de la Revolución (Museo Jacobo Borges); Exposición Temática sobre el 4-F (Museo Alejandro Otero), entre otros. Esto nos permite entender la visión instrumental de la cultura del gobierno actual que no en balde habla de "ética y estética socialistas".

\section{El hombre nuevo, la mujer nueva: objetivos estratégicos de la Revolución Bolivariana}

El Gobierno cuenta con creadores reconocidos de diversas disciplinas que lo respaldan, con una trayectoria y una obra personal importante; igualmente, tiene gente joven que está fraguando sus propuestas personales. Pero más allá de su calidad, los artistas, escritores y cultores no han logrado del todo el salto creativo de la poesía, el cine y los afiches soviéticos de los primeros tiempos de la revolución bolchevique. Tampoco este período de revolución ha sido testigo de esa audacia creativa que alimentó la trova cubana y el muralismo mexicano, que se convirtieron en banderas culturales de sus países y expresión de un estado de revolución social. Se registran como "novedades" infrecuentes para 1999 poemas de veinteañeros que hacen odas a Chávez (algunos pertenecientes a poetas jóvenes muy prometedores, por cierto), imitadores de Alí Primera que hacen "canción 
necesaria", jóvenes locutores que hablan de la revolución cultural china en las radios comunitarias, murales y grafitis financiados por el Estado: puro pasado, una moda nostálgica de los años sesenta con su toque indígena, su toque afro y su toque Che Guevara. Mucho más atractivos son los colectivos como Tiuna El Fuerte que en vídeos como "Petare Será otro Beta" demuestran una labor de animación cultural en los barrios que recoge bailes populares, hip hop y deportes como el boxeo y el básquet, en lugar de imponer una estética pasatista que no le dice nada a los sectores populares que se supone son protagonistas de la revolución. Igualmente, los registros de estéticas urbanas juveniles internacionalizadas que se basan en la exaltación de los barrios y la vida de ciudad, presentes por ejemplo en Ávila TV, son más interesantes que esa instrumentación política tan burda de las exposiciones en los museos sobre el 4 de febrero.

Mucho más visible y exitosa que revivir estéticas del pasado o hacer estas manipulaciones políticas del arte, ha sido la instrumentación propagandística del Sistema Nacional de Orquestas y Coros Infantiles y Juveniles, con Gustavo Dudamel, extraordinario director de orquesta que presta sus servicios en actos del Gobierno nacional, y con las giras de la orquesta por todo el mundo: el socialismo convertido en música. La verdad es que el sistema se fundó hace casi cuarenta años, contó con el apoyo de todos los Gobiernos y el Maestro José Antonio Abreu, cuya obra sin duda quedará para la historia y debe ser orgullo de la Venezuela en democracia en el futuro, fue Presidente del Consejo Nacional de la Cultura cuando gobernaba el denostado Carlos Andrés Pérez, cuyo Gobierno fue objeto de los dos golpes de Estado del año 1992.

Ahora bien, según el actual Ministro del Poder Popular para la Cultura Fidel Barbarito, la estética socialista recogerá y expresará los valores del hombre y la mujer nuevos. ¿Cuáles son estos valores que deberían sustentar las creencias, los modos de vida y la legitimidad de las prácticas culturales en el país? Veamos los siguientes objetivos estratégicos que formula el Proyecto Nacional Simón Bolívar Primer Plan Socialista (2007-2013):

I. Nueva ética socialista: Propone la refundación de la Nación Venezolana, la cual hunde sus raíces en la fusión de los valores y principios más avanzados de las corrientes humanistas del socialismo y de la herencia histórica del pensamiento de Simón Bolívar.

II. La suprema felicidad social: A partir de la construcción de una estructura social incluyente, un nuevo modelo social, productivo, humanista y endógeno, se persigue que todos vivamos en similares condiciones, rumbo a lo que decía El Libertador: "La Suprema Felicidad Social". 
Estos objetivos persiguen la construcción de una nueva hegemonía término proveniente del pensador marxista Antonio Gramsci que implica la constitución de una cultura sustentada en valores alternativos a los heredados de las sociedades capitalistas-, palabra frecuentemente mencionada por los ministros, por el actual presidente y en vida por Hugo Chávez Frías. En este orden de ideas, las políticas culturales son fundamentales en la conformación del hombre y la mujer nuevos que dejarán atrás los valores propios de la desigual, explotadora e injusta sociedad capitalista. El Estado revolucionario pretende tener decisión sobre la manera en que los hombres y mujeres en Venezuela nos vemos a nosotros mismos como pertenecientes a un territorio determinado definido en tanto comunidad de destino: existimos bajo un ordenamiento legal que condiciona nuestros actos, y somos parte de una economía que modela, limita o estimula estilos de vida; comemos, respiramos, amamos y nos entendemos dentro del conjunto de prácticas sociales y simbólicas reconocidas como propias de la región o de la nación dados el sistema educativo, las tradiciones familiares y locales. Precisamente en este tejido vital y entrañable la lógica revolucionaria pretende intervenir. A pesar de que la Constitución de la República Bolivariana de Venezuela consagra la naturaleza pluricultural del estado venezolano, tal pluriculturalidad se acepta en términos de la diversidad cultural regional y de las culturas constitutivas de la nacionalidad, en especial las de origen claramente indígena o afro-venezolano, pero no en términos de los procesos de hibridación cultural e interculturalidad que han ocurrido en el país a lo largo de su historia.

La modernidad venezolana que se ha producido a través de las migraciones, la educación masiva, la explotación petrolera y el crecimiento del Estado, los grandes rasgos que definen nuestra existencia nacional, es claramente conceptuada desde la revolución como un error que ha permitido el desarrollo de una serie de prácticas racistas, consumistas, alienantes, elitistas y pro-imperialistas que han penetrado desde la forma de comer hasta el vestir, pasando por las universidades, los museos, el cine, la sexualidad, la literatura y los medios de comunicación. La obsesión de organizar una institucionalidad paralela en todos los terrenos de la acción del Estado y de dividir desde las familias, los sindicatos y las iglesias hasta las organizaciones políticas, culturales y empresariales, obedece a este voluntarismo revolucionario puritano, ansioso de salvar de sus pecados a los paganos del culto al capitalismo yanqui.

Luego de catorce años de Gobierno, la mitad de la población se resiste a aceptar las nuevas reglas del juego de sometimiento absoluto al Estado a pesar de los cuantiosos recursos invertidos en políticas de comunicación, cultura y educación orientadas al cumplimiento de los fines del Estado revolucionario. Esta 
resistencia obedece a esa modernidad rentista que convirtió en cultura popular las aspiraciones de autonomía y logro personal así sea en el terreno de los deseos más que en el de las realizaciones: hijos de los flujos migratorios, la educación masiva aunque precaria, la cultura popular internacional y el discurso del igualitarismo y el heroísmo independentista, los hombres y mujeres opositores al Gobierno, y suponemos que una parte nada despreciable de sus seguidores no se adaptan a la idea de una cultura nacional, una "identidad nacional" auténtica orquestada y rescatada desde el Estado. Desde luego, los errores de gestión no ayudan al Gobierno nacional en este sentido ni el empeño propagandístico tampoco, pero este trasunto cultural moderno que, por cierto, no idealizamos en lo más mínimo sino que simplemente registramos desde los terrenos de la investigación cultural, es vital para entender lo que ocurre en Venezuela respecto a los resultados de las políticas culturales, comunicacionales y educativas del Estado.

\section{¿Se han saldado las deudas históricas del estado con el sector cultural?}

En las Memoria y Cuenta del Ministerio del Poder Popular para la Cultura se reconoce la ineficacia en cuanto a gestión propiamente dicha: no ejecución de proyectos presupuestados, deficiente plataforma tecnológica, falta de personal calificado (en un país que cuenta con graduados suficientes para cubrir esta demanda) y problemas con la importación oportuna de insumos, fenómenos relacionados con la actuación interventora y obstaculizadora que caracteriza al estado venezolano hoy. En su estrategia de planificación centralizada inspirada en los socialismos reales del siglo XX, la revolución bolivariana ha sustraído competencias a alcaldías y gobernaciones, en especial a las conducidas por opositores, con el consiguiente debilitamiento presupuestario de las mismas y la reducción de su capacidad de gestión. La presencia de las plataformas culturales en los Estados, municipios y consejos comunales así como de los gabinetes estadales y la Misión Cultura supondría una fuerte desconcentración y descentralización de la gestión, una deuda con el sector para 1999, si no estuviera mediada por el sectarismo político desde el punto de vista del contenido de la programación cultural, los gestores cuadrados con la revolución y la discrecionalidad política en cuanto a los destinatarios de los recursos.

Sin duda alguna, este Gobierno ha colocado a los sectores populares urbanos y rurales como el norte de sus políticas públicas y desde el punto de vista simbólico ha logrado que el país entero entienda su protagonismo en la Venezuela del siglo XXI. El problema es que nada se salva de la voracidad autoritaria de la revolución: tanto la Misión Cultura, con muy fuerte presencia cubana, como el Sistema Nacional de Culturas Populares exigen adhesión al Plan Socialista 
Simón Bolívar: "Son miembros del Sistema Nacional de las Culturas Populares todas las instituciones públicas y privadas relacionadas con gestión cultural que manifiesten ante el órgano rector su voluntad de pertenecer al Sistema y que acepten como suyas las líneas estratégicas del Proyecto Nacional Simón Bolívar".

Artista, cultor o gestor que no apoye explícitamente al oficialismo queda fuera del sistema, lo cual obliga a la auto-censura, el silencio o la adhesión obligada en franco irrespeto a principios constitucionales como la libertad de expresión y pensamiento, la libertad de creación y el pluralismo político. En conclusión, la deuda cultural con los sectores de menores recursos por lo visto pretende ser pagada con chantajes de por medio. Además, si bien es perfectamente plausible que se promocione, pongamos por caso, un instrumento como el cuatro en igualdad de condiciones con el violín o el piano, entender como cultura nacional fundamentalmente la relativa a la tradición, sesgo evidente como ya se dijo en el Proyecto de Ley Orgánica de Cultura a aprobar este año 2013, no contempla la naturaleza de la cultura en el siglo XXI y entra incluso en contradicción con las políticas de Estado relativas al uso de las tecnologías de información y comunicación en el cambio social, educativo, político y cultural. De la misma manera, el intervencionismo estatal debilita la autonomía de artistas, cultores y gestores pues en lugar de manejarse en términos de emprendedores culturales lo hacen en términos de subsidiados por el Estado. El Estado, se debe insistir hasta la saciedad, debe acompañar la creación cultural, no condicionarla de ningún modo.

Estos prejuicios contra la modernidad cultural se evidencian también en el prurito absurdo respecto a hablar de las industrias culturales por considerar este término como manifestación del capitalismo y sinónimo de Hollywood. La producción gráfica y cinematográfica, la radio y televisión de servicio público, el diseño, el turismo cultural, son industrias independientemente de que se trate de la Villa del Cine o de los estudios Warner en Estados Unidos. Este resabio anti-moderno se manifiesta igualmente en el rechazo a la dimensión económica de la cultura, vista sólo en términos de inversión social (efectivamente lo es y hay que darle la importancia y el presupuesto que merece), y no como la posibilidad de crear empleo y prosperidad a través del emprendimiento y el desarrollo cultural. Este corsé ideológico se hace presente en otra deuda gigantesca de las políticas culturales venezolanas: el rol cultural de la radio y televisión de servicio público, su labor como tribuna preferente para dar a conocer los valores y la memoria nacionales del pasado y del presente sin distingo político. Las numerosas radios comunitarias y los canales de televisión como Ávila TV, Vive TV, Colombeia, VTV, son tribunas del oficialismo, más allá de los programas de carácter cultural que puedan eventualmente transmitir, entre los que, además, no faltan los documentales y reportajes 
que monótonamente tratan de inculcar las visiones políticas promocionadas por el Gobierno; la orientación ideológica no se disimula ni el carácter militante de quienes producen y llevan los programas en vivo, tampoco. El absurdo argumento de que hay que hacerle contrapeso a las "emisoras de la derecha" olvida que éstas se financian con dineros privados y exhibe el radical autoritarismo al que estamos sometidos en Venezuela, pues se usan los recursos de todos para desconocer a la mitad del país que no comulga con el proyecto revolucionario. Además, tienen baja audiencia porque, al igual que en otras áreas de la gestión cultural, se invierte dinero pero parece importar poco el impacto poblacional real.

Por supuesto, dados los esfuerzos proselitistas del Gobierno en el marco de un país polarizado políticamente como Venezuela, su empeño en hablar de la cultura venezolana en términos de cultura o "identidad nacional" no sólo es discutible a estas alturas del siglo XXI, dados los debates sobre estos temas en el contexto de la globalización, sino que además causa resistencia y suspicacia. Por una parte, en Venezuela, como en todos los Estados nacionales, existen multitudes de prácticas culturales y diversos "sistemas de valores" que funcionan para determinados sectores y no para otros, independientemente de que compartan un territorio y un ordenamiento jurídico y político determinado. La revolución miente la verdad: si bien la exclusión social y económica en medio de la prosperidad petrolera fue muy real, sobre todo a partir de los años ochenta del siglo pasado, no es cierto que esta lógica articuló todos los espacios de la vida intelectual venezolana y se expresó en una cultura racista, acartonada, burguesa y antipopular que, por lo tanto, hay que purificar sistemáticamente. La deuda del Estado con la diversidad esencial de la sociedad venezolana sigue vigente pues la exclusión relativa de lo popular urbano, rural y aborigen no puede ser saldada con el sistemático ataque a otras herencias, registros culturales, formas de pensar y crear y, sobre todo, no puede pagarse a espaldas de la pertenencia de Venezuela al concierto internacional, no sólo caribeño y latinoamericano, sino mundial. Cuando volvamos a la democracia, será precisamente el reconocimiento de esta diversidad esencial en el contexto de un mundo globalizado elemento central de la reconstrucción del tejido social, económico político y cultural de la nación venezolana.

\section{Obras citadas}

Boon, Lisseth y Jesús Alberto Yajure. "Venezolanos Comelibros". Últimas

Noticias 24 de marzo de 2013 Suplemento 'El Domingo': 1-3.

Centro Nacional del Libro. Estudio del Comportamiento Lector, Acceso al Libro

y la Lectura en Venezuela 2012. Caracas: Centro Nacional del Libro, 2012. 
Delgado Flores, Carlos. "El Libro: Entre Cuentos y Cuentas". SIC 2013: 41-43.

Instituto de Investigaciones Económicas y Sociales de la Universidad Católica Andrés

Bello. "Estudio de Consumo Cultural en Venezuela 2010". SIC 23 de marzo de 2010.

Ministerio del Poder Popular Para la Cultura. Sistema Nacional de las Culturas

Populares. Caracas: Ministerio del Poder Popular Para la Cultura, 2011.

Proyecto Nacional Simón Bolívar-Primer Plan Socialista-PPS-2007-2013. 29

Marzo 2011. Caracas: Partido Socialista Unido de Venezuela, 2007.

Ramos, María Elena. La Cultura Bajo Acoso. Caracas: Artesano Editores, 2012.

UNESCO. Convención Sobre la Protección del Patrimonio

Mundial, Cultural y Natural. París: UNESCO, 1972.

-. Recomendación sobre la normalización internacional de las estadísticas relativas al financiamiento público de las actividades culturales. París: UNESCO, 1980.

-. Declaración de México sobre Políticas Culturales. París: UNESCO, 1982.

-. Convención sobre la protección y promoción de la diversidad de las expresiones culturales. París: UNESCO, 2005. 